\title{
HACIA UN MODELO SISTÉMICO-TRANSACCIONAL DE ANÁLISIS E INTERVENCIÓN SOCIAL
}

\section{Alberto Saco*}

\section{INTRODUCCIÓN}

El enfoque sistémico de la sociedad elaborado por Talcott Parsons (1959/ $1966 ; 1961 / 1968)$ ha sido muy criticado tanto por su 'grandiosa' complejidad (Mills, 1959/87 44 ss.) como por sus consecuencias, aparentemente conservadoras. Si bien el autor trata de 'rescatar' al individuo para el análisis social, en una línea explícitamente weberiana (Parsons, 1959/1966), el hecho es que acaba por 'encerrar' al individuo en el estrecho margen de un sistema cultural que presupone con una existencia objetiva, algo que está más en la línea defendida por Durkheim ${ }^{1}$. Luhmann (1996: 35 ss.) también critica a Parsons la cerrazón de su esquema teórico, que da al sistema social una variedad limitada de posibilidades de acción.

Ello no debería, sin embargo, hacernos renegar del valiente intento del autor de conciliar o poner en relación plausible individuo y sociedad, acción y estructura social. Es más, en la última década asistimos a un resurgir de la línea weberiana de análisis. El individuo y la acción individual vuelven a ocupar un lugar importante en el análisis de la sociedad y de la acción social. La demostración más fehaciente de ello es el éxito cosechado por las teorías de la decisión racional, aplicadas primero en el terreno de la economía, en el intento de devolver al individuo el protagonismo de la acción social. Así, primero Olson (1971/1992) y después Axelrod (1984/1986) tratan de explicar como es posible la acción colectiva desde el supuesto común de que los que actúan son individuos y que lo hacen racionalmente.

\footnotetext{
* Profesor de Sociología de la Universidad de Vigo
} 
Olson da una solución al dilema del 'free rider'" basado en la administración de los incentivos selectivos por parte de una autoridad que sanciona a quien defrauda la norma del grupo. Axelrod va más allá y explica cómo es posible la cooperación sin que sea necesaria la aplicación de sanciones por una autoridad. Cuanto más se prolonga la interacción entre individuos más probable es que éstos lleguen a la conclusión de que lo más racional para ellos es cooperar en lugar de enfrentarse o sacar provecho unos de otros. El problema que, a mi entender, presentan dichas teorías de la acción social es que presuponen una racionalidad (probada, por otra parte) a las acciones individuales cuando sabemos que no siempre nos comportamos racionalmente. Parece demostrado que los seres humanos tenemos serias limitaciones internas y externas para comportarnos de una manera totalmente racional ${ }^{3}$. Limitaciones internas derivadas de nuestra estructura de personalidad y limitaciones externas derivadas de la estructura social, que, entre otras cosas limita los flujos de información a los que tenemos acceso en función de nuestra posición en la red social. Luego, ni gozamos de la objetividad en la toma de decisiones, ya que en éstas entran en juego componentes no-racionales, ni disponemos de toda la información para tomar las más adecuadas. La jaula de hierro de la racionalidad no acaba de casar con nuestra naturaleza humana, limitada.

Por eso se hace necesario crear un marco teórico adecuado para reflejar las limitaciones internas y externas de la acción individual y poder medir sus consecuencias para el sistema social. Las limitaciones internas pueden ser abordadas desde el paradigma psicológico del análisis transaccional de Eric Berne (1961/ 1976 $)^{4}$, mientras que las externas estarían en relación con las teorías de redes sociales aplicadas por algunos antropólogos en sociedades complejas (Mayer, 1966/ 1990). Lo que se pretende, en el fondo, es matizar, dentro de un enfoque sistémico, las teorías psicoanalíticas de Freud con las transaccionales de Berne, que suman a una mayor simplicidad su capacidad para explicar los mecanismos de interacción social en función de la estructura de personalidad de los individuos.

El resultado sería una teoría sistémica de fácil aplicación para el análisis y la intervención sociales que concilia el sistema-individuo con el sistema social y la acción social con la estructura. Esta teoría constaría de un modelo homeostático con tres niveles de análisis e intervención atravesados por tres sistemas isomórficos o con características comunes entre ellos.

\section{ELEMENTOS DEL MODELO}

Los niveles de análisis en Sociología ya fueron planteados en su día por Gurvitch (1964). Ritzer (1979) retoma el tema de la integración de estos niveles, planteando la existencia de un nivel micro y otro macro, ambos dotados con una doble naturaleza, objetiva y subjetiva.

Para nuestro modelo, consideramos que existen tres niveles de análisis del 
sistema social, en función del tamaño del colectivo y el grado de formalización de las relaciones entre individuos. El primero, micro o grupal, que abarca a los fenómenos sociales que tienen lugar entre individuos dentro de los grupos sociales. Un segundo nivel, meso u organizacional, referido al análisis de lo social dentro de la esfera organizativa. Un tercer nivel, macro o institucional, que hace referencia a la sociedad en su conjunto (ver figura 1).

Figura 1. -Los tres niveles de análisis del sistema social

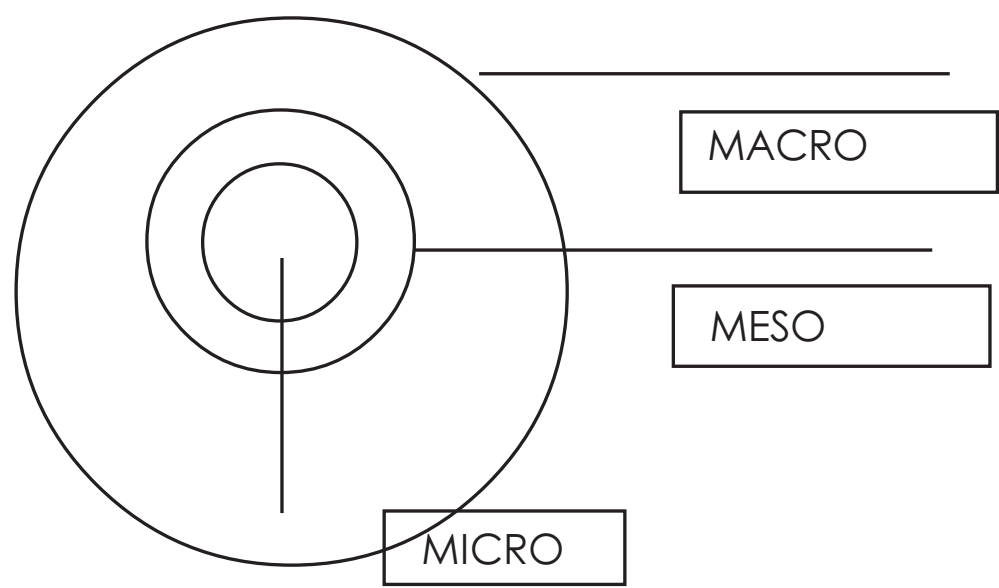

Es de vital importancia explicitar que se trata de favorecer, parafraseando a Durkheim (1894/1982), el análisis de lo social desde lo social. No se trata de trasladar al sistema social las características de los individuos que lo conforman, cayendo en un enfoque psicologista, sino, por el contrario de considerar la conexión existente entre individuos con características psicológicas diferentes como la clave para entender el entramado de relaciones sociales que configura la realidad social. Para ello es que se considera como objeto de estudio la interacción social en cada contexto o nivel, teniendo como unidad mínima verificable la transacción ${ }^{5}$. La transacción sería la unidad de relación social, consistente en un proceso constatable de acción-reacción entre, al menos, dos individuos. Las transacciones que llevan a cabo los individuos tienen lugar en estros tres niveles dentro de tres sistemas isomórficos o que comparten unas ciertas características: el sistema individuo, el sistema transaccional y el sistema social. Los tres sistemas presentan una estructura tripartita o con tres niveles que hacen que los procesos que tienen lugar entre ellos en uno sean (formalmente) equiparables en cualquiera de los otros y además estén conectados por mecanismos que transmiten información de un sistema a otro atravesando los tres niveles del sistema social. En los tres sistemas se pueden diferenciar tres partes que intervienen en las esferas normativa, instrumental y expresiva. 
El sistema individuo explica las limitaciones internas a la interacción, el sistema social, las externas y el sistema de interacción, los conjuntos de acción colectiva o mecanismos que los individuos ponemos en marcha al tratar de maximizar nuestras acciones dentro de las limitaciones y potencialidades de ambos sistemas.

\section{EL SISTEMA-INDIVIDUO}

Los individuos no interactúan en el vacio, ni interior ni exterior. Cada individuo posee una estructura de personalidad con elementos comunes a otros, pero también con elementos únicos e irrepetibles. Toda acción social es significativa en términos individuales o de motivación íntima del sujeto y en términos sociales o de comunicación. Sobre la significación social de la acción individual habrá que referirse más tarde al sistema social. Para explicar la significación subjetiva de la acción, es necesario remitirse a la propia estructura interna del sistema-individuo como motivadora, limitadora y potenciadora de sus acciones. Motivadora, porque es en el individuo donde residen las necesidades, deseos y pulsiones personales e intransferibles que le mueven a actuar. Limitadora, porque el individuo ha interiorizado (más o menos) a través de su proceso de socialización la forma de expresar sus pulsiones adecuada a la norma social. Potenciadora, porque el individuo tiene un cierto margen de decisión sobre hasta que punto plegarse o no a la norma en función de sus expectativas de éxito o fracaso. En otras palabras, puede comportarse racionalmente y decidir qué es lo que más le conviene. Estas tres características de la estructura de personalidad de los individuos son trasladables a los otros dos sistemas o estructuras. En el caso del sistema-individuo y, siguiendo el modelo del análisis transaccional, se corresponderían con el niño, el padre y el adulto, respectivamente.

El niño es el elemento no-racional de nuestra personalidad que busca la satisfacción inmediata de sus necesidades y deseos, nuestra parte orgánico-biológica, pendiente de la supervivencia, la reproducción o la defensa del territorio entre otras cuestiones. Es el id reflejado en el psicoanálisis. En el residen los fines y las motivaciones. En él reside la función expresiva del sistema-individuo.

El padre es también una parte no-racional, aquella que consiste en el bagaje normativo que hemos interiorizado a través del proceso de socialización. Sería el super-ego, en la terminología freudiana. En él residen los principios y los límites, o sea, la función normativa.

Finalmente, el ego o el adulto, trata de conciliar realidad externa con bagaje normativo y deseos para lograr un cierto equilibrio. Sería la parte centrada en los medios y en planificar y evaluar los resultados de la acción. En el adulto se sitúa la función instrumental del sistema-individuo.

En este proceso de búsqueda de equilibrio interno y con el exterior, el individuo exterioriza o envía outputs ${ }^{6}$ provenientes de cada uno de sus elementos, 
es decir, se relaciona con su entorno (natural y social) desde el niño, el padre o el adulto (ver figura 2). Cada transacción social realizada pone en juego, bien los deseos, las normas o las ofertas y demandas de cada individuo. El tipo de transacción desarrollado entre individuos conformará en cierta forma el entramado de relaciones dentro del grupo, la organización o el sistema social en que tengan lugar.

Figura 2. - Estructura interna del sistema-individuo

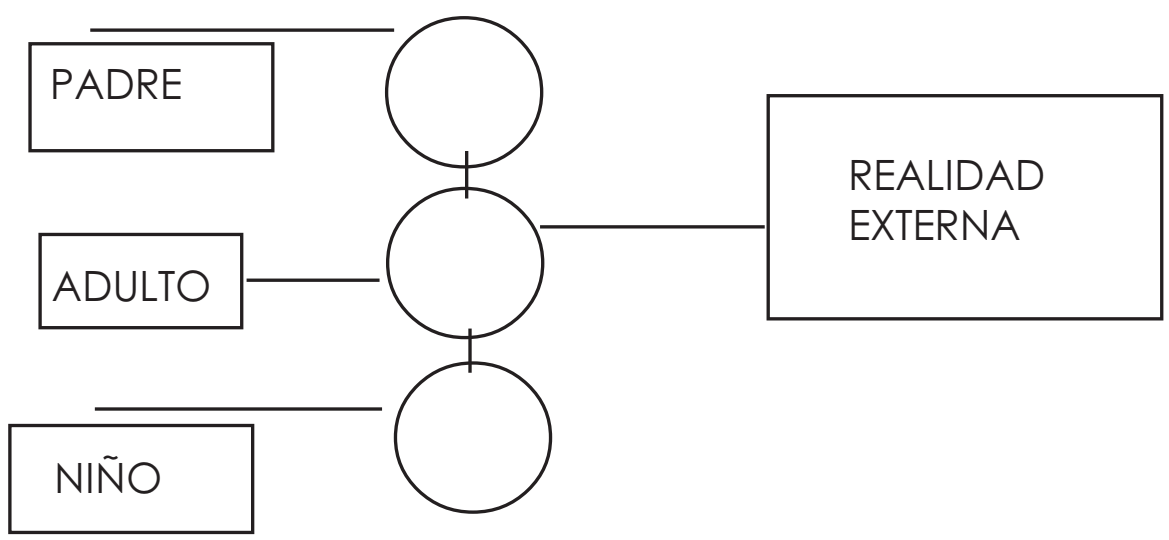

Partimos del supuesto de que los individuos tienden a comportarse de una manera racional, a jugar juegos de suma 1, en los que todos ganan. Esto es cierto en algunos tipos de transacción o de interacción, como son las relaciones de conformidad, intercambio y complicidad. En todos los casos, los individuos salen ganando con la interacción y ésta tiende a continuar, generando a través de rutinas estabilidad y estructuración social (Giddens, 1984).

La relación de conformidad viene dada por las transacciones de padre a padre (P-P), la de intercambio por las desarrolladas entre adulto y adulto (A-A) y la complicidad por aquellas que desempeñan los niños de los individuos (N-N). Un predominio de las transacciones de conformidad configura rutinas y contextos sociales de tipo tradicional. La prevalencia de transacciones de complicidad genera contextos sociales carismáticos. La mayor importancia de transacciones de intercambio genera contextos de corte legal-racional.

Sin embargo, los individuos no siempre ganan en las transacciones, y existen formas de transacción que tienden a permanecer en el tiempo y que consisten en juegos de suma $\mathrm{O}$, en los que un jugador gana y otro pierde. Sería el caso de las relaciones de sumisión, basadas en la coerción que un individuo ejerce sobre otro. En este caso, la expectativa de poder perder más aún, hace que un individuo se someta a otro y perciba la pérdida como una ganancia relativa. La prevalencia de este tipo de transacción, padre-niño (P-N), conforma contextos sociales paternalistas y/o autoritarios. 
Estos cuatro tipos de transacción, denominadas complementarias, porque son recíprocas y tienden a continuar en el tiempo tienen por ello un gran poder de estructuración social en la medida en que derivan en rutinas y en contextos sociales a cualquier nivel. Serían tipos 'puros' o ideales, puesto que existen otras transacciones mixtas o de transición (padre-adulto, niño-adulto) que no van a ser tratadas aquí pero que serían clave para explicar los mecanismos de mediación social (ver figura 3).

Hay otras formas de transacción social que suponen la interrupción de la comunicación y que reciben la denominación de 'transacciones cruzadas'. La más típica es la padre- niño/padre-niño (P-N/P-N) en la que ambos individuos se recriminan mutuamente sus pretensiones en función de su sistema de valores. Se trata de una relación de conflicto, con poca capacidad para generar estructura pero con mucha para destruirla o interrumpir los flujos transaccionales.

Figura 3. - Tipos principales de transacción

\section{COMPLEMENTARIAS}

Igualitarias: Suma 1
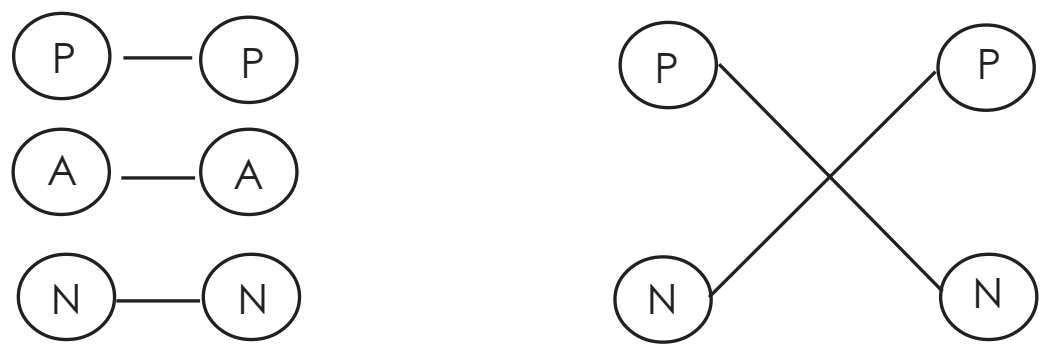

Desigualitarias: Suma 0

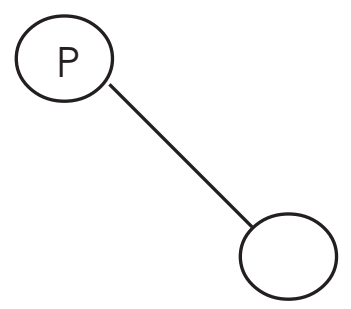

Las transacciones sociales no son sólo una expresión de los outputs de los individuos sino que a su vez generan outputs de un tipo u otro hacia el sistema social a través del sistema transaccional o la red social. Tienen lugar pues en el 
contexto limitado de la estructura de personalidad de los individuos y de la red social (o sistema transaccional) en la que interactúan desde una determinada posición social.

\section{EL SISTEMA TRANSACCIONAL}

El sistema transaccional consiste en el entramado de transacciones sociales previamente estructurado en función de posiciones sociales reconocidas. Presenta también una estructura tripartita que motiva, limita y potencia la acción social. Es el equivalente a la red social, compuesta de focos, mediadores y líderes que representan a los distintos sectores de base (ver figura 4).

Figura 4. - Estructura interna del sistema transaccional

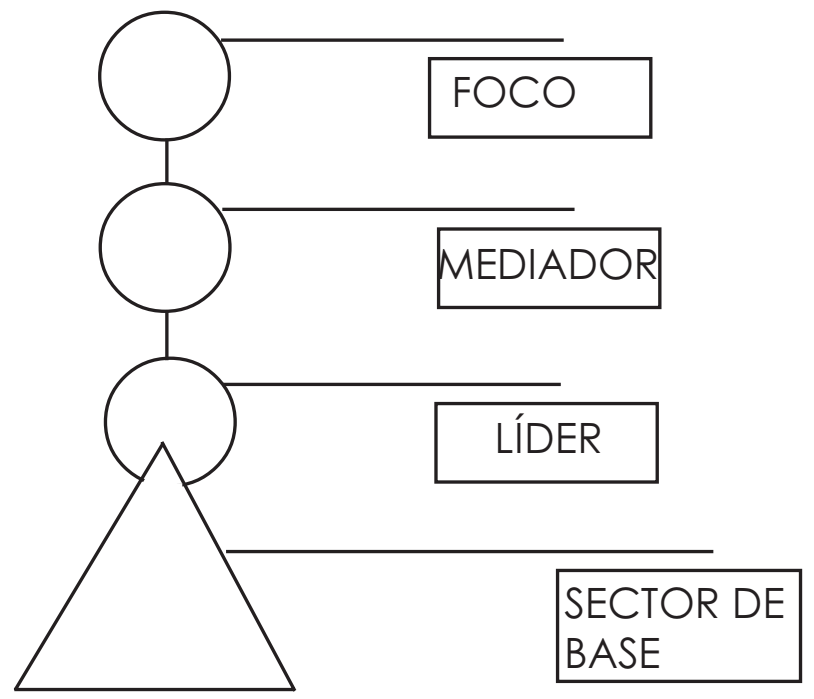

Es necesario aclarar que la consideración de la red social como un sistema en sí mismo depende en gran medida del nivel de autopoiesis y de diferenciación con el entorno (Luhmann, 1984/1998) alcanzado por cada red. En la mayoría de los casos, las redes funcionan como cuasi-grupos (Mayer, 1966/1990), no presentando el nivel de autoconciencia y de diferenciación con el entorno que permitirían considerarlas como un sistema. En tales casos, la red social constituiría el mecanismo mediante el cual se ponen en conexión los sistemas individuo y social, intercambiando flujos de información. La red social puede ser pues considerada bien como un sistema, bien como un mecanismo que vehicula los outputs de los dos sistemas citados. 
La posición de los individuos en la red social influye en su toma de decisiones, de la misma forma en que lo hace su estructura de personalidad, pero a otro nivel. También su estructura de personalidad influye en la posición social ocupada, ya que las instituciones, organizaciones y grupos realizan algún tipo de selección entre sus miembros para desempeñar los papeles acordes con las distintas posiciones sociales (Gerth y Mills, 1984).

La posición social y el rol de líder están íntimamente relacionados con la motivación para la acción. El líder, en mayor medida que los otros componentes de la red, estimula y expresa los deseos, necesidades y temores de los sectores de base que representa. Está por lo tanto, más marcado en el plano de lo social por el carisma y en el plano individual por una mayor prevalencia del componente niño. Está más en la esfera del querer que en la del saber o la del poder. En él reside en mayor medida la función expresiva dentro del sistema transaccional.

El estatus y el rol de mediador conllevan un mayor peso del componente racional (o adulto), sometiendo en mayor medida que los otros agentes sociales sus acciones a cálculo, medición y control. Se mueve en la esfera del saber con más frecuencia que las otras dos posiciones, potenciando tanto al poder como al querer. En el mediador tiene mayor peso la función instrumental.

La posición de foco implica un mayor componente normativo y sancionador (o padre), manifestándose claramente en la esfera del poder (económico, cultural o político) y en el desempeño de la función normativa.

Las tres posiciones se relacionan entre sí de manera estable a través de transacciones complementarias. Como en el sistema-individuo, la relación inmediata es la que tiene lugar entre padre y niño, entre deseo y norma, entre querer y poder, por lo que se buscan transacciones complementarias entre estas dos posiciones. La intervención del mediador introduce una mayor racionalidad a los procesos de toma de decisiones en cuanto suministra y demanda información de unos y otros para introducirla en el proceso. De no haber mediadores, la transacción complementaria más frecuente entre foco y líder es la de sumisión, generando contextos sociales paternalistas o conjuntos de acción de corte populista. La presencia del mediador social (miembro de una elite tecnocrática) hace posibles otros tipos de transacciones entre foco y mediador y entre líder y mediador, marcadas por un mayor componente racional (adulto). Esto puede conducir, bien a una mayor densidad de flujos transaccionales entre foco y mediador, dando como resultado un contexto social de control burocrático o un conjunto de acción gestionista, o bien a una mayor densidad de transacciones complementarias entre mediador y líderes, resultando un contexto social de cambio revolucionario. Una situación óptima de equilibrio entre flujos transaccionales conduciría a un contexto de reforma democrática o a un conjunto de acción ciudadano.

La ventaja que ofrece un enfoque transaccional es que nos permitiría medir, desde el papel de técnico o mediador, dichos flujos transaccionales, contemplando no sólo menor o mayor cantidad de información compartida sino también el tipo de interacción desarrollada. 
En un conjunto de acción populista, prevalecerían las transacciones complementarias P-N. En un conjunto de acción gestionista, las transacciones P-A. En un conjunto de acción ciudadano, las transacciones A-A. En un proceso de reforma revolucionaria, las transacciones predominantes serían las de A-N, tras un estadio previo de fervor revolucionario dominado por las transacciones N-N.

\section{EL SISTEMA SOCIAL.}

El sistema social consistiría en el entramado de relaciones sociales más o menos estables (o estructuradas) entre un conjunto de individuos y/o colectivos. Estas pueden afectar a un colectivo más o menos grande y estar revestidas de una mayor o menor formalización. Podemos distinguir los tres niveles antes citados. El micro o grupal, el macro o institucional y el meso u organizacional. Los tres están conectados mediante redes sociales, que hunden sus raíces en el sistema individuo y el nivel micro y expanden sus ramificaciones en el sistema social hasta el nivel macro (ver figura 5).

El sistema social pues recibiría outputs ascendentes de los sistemas-individuo y transaccional y enviaría a su vez outputs descendentes a través del sistema transaccional o red social.

Un sistema social analizado en su nivel micro (un grupo) estaría estructurado en función de las transacciones entre individuos que tienen lugar dentro del mismo y de los outputs recibidos desde arriba. Estos outputs son recibidos en el grupo a través de aquellos individuos que ocupan una posición social de liderazgo en el grupo. Asimismo, el líder, exterioriza y envía hacia arriba los outputs resultantes a nivel grupal o micro. En el nivel micro-social tendría más peso la función expresiva.

Figura 5. - Los tres niveles de análisis e intervención y el sistema transaccional.

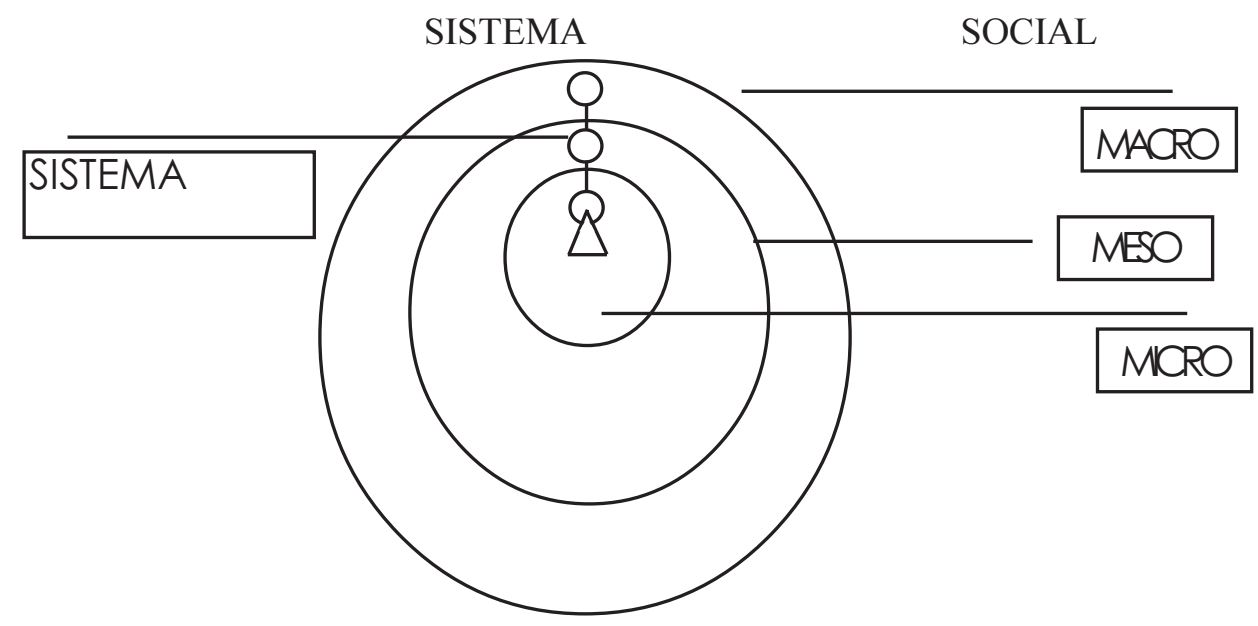


El nivel meso hace referencia al entramado organizacional de la sociedad, formado por las organizaciones formales e influenciado por los outputs ascendentes del nivel micro o grupal y por los outputs descendentes del nivel macro o institucional. Los mediadores son el elemento del sistema de interacción que vehiculan dicha información, trasladando las demandas desde los grupos hasta la organización y desde ésta al nivel institucional a través de transacciones complementarias sucesivas entre líder y mediador (N-A) y mediador y foco (A-P). De no existir mediación social o, lo que es lo mismo, de carecer el sistema social de un tejido organizativo más o menos tupido y operativo, las transacciones tienden a ser inmediatas y complementarias entre líderes y foco $(\mathrm{P}-\mathrm{N})$, consolidándose una estructura paternalista y/o autoritaria de la acción social. Cuando el mediador desarrolla su tarea más de cara al foco que de cara a los líderes se consolida una estructura burocrática (basada en la racionalidad instrumental), de la acción social. Cuando el mediador transacciona preferentemente con los líderes de los sectores de base se genera una estructura revolucionaria (basada en una racionalidad expresiva), generalmente enfrentada a la norma. En general, el crecimiento del tejido organizacional introduce una mayor racionalidad a la acción social. La burocratización y la racionalización del mundo parece desarrollarse en este sentido. En el nivel meso reside en mayor medida la función instrumental del sistema social.

El nivel macro comprende todas aquellas acciones sometidas a un sistema de sanciones altamente formalizado o altamente institucionalizadas. En él están situadas en mayor medida que en los otros las funciones normativas del sistema social. Las instituciones familiares, políticas, religiosas, económicas o militares atravesarían con sus normas o pautas de orientación todo el sistema social de arriba abajo, bien a través de los mediadores, bien directamente a través de los líderes. El papel de los mediadores o de la mediación social ha ganado importancia en la medida en que las organizaciones formales adquieren más importancia en nuestras vidas. En una sociedad de corte tradicional, la institución incide de manera inmediata en los grupos sociales. Un buen ejemplo de esta relación inmediata es el grupo familiar, atravesado por las instituciones del matrimonio y la filiación, aunque hasta este, hasta ahora, coto vedado a la mediación social va siendo afectado en mayor medida por flujos transaccionales que provienen del tejido técnico-organizativo más que del tejido 'moral' de la sociedad.

Un sistema social de corte tradicional presupone la prevalencia de transacciones del tipo P-P. Una sociedad de corte moderno sería el resultado del crecimiento del tejido organizativo y el consiguiente incremento de las transacciones de tipo A-A. Una sociedad en estado naciente se caracterizaría por la prevalencia de transacciones N-N. Una sociedad autoritaria estaría configurada en su mayor parte por transacciones P-N. Finalmente, una sociedad en conflicto abierto o en choque cultural con otro sistema social tendría un entramado de transacciones excluyentes predominantemente del tipo P-N/P-N. 
Habría también tipos mixtos o de transición. La prevalencia de transacciones del tipo P-A configurarían una sociedad de corte burocrático, la de las A-N, una sociedad de corte revolucionario.

Como se puede atisbar, los flujos transaccionales configuran sistemas de alianzas entre elites tradicionales, tecnocráticas y carismáticas, dando lugar a distintas estructuras sociales con claras consecuencias para la vida política, como ya argumentara en su día Barrington Moore (1976). Los flujos transaccionales más frecuentes en las elites tradicionales son del tipo P-P, mientras que las elites tecnocráticas desarrollan en mayor medida transacciones del tipo A-A. Las elites carismáticas, por el contrario, promueven en mayor medida las transacciones N-N.

\section{ESTRUCTURA Y CAMBIO SOCIAL}

A continuación se tratará de realizar una sinopsis de las formas de estructuración y de cambio social resultantes de los flujos transaccionales predominantes en un sistema social en cualquiera de los niveles contemplados.

En cuanto a las estructuras sociales o contextos rutinizados para la acción social, se considerarán básicamente los tipos ideales o en estado puro, para después pasar a explicitar la relación que guardan con los tipos de transición, generalmente resultantes de procesos de vertebración y racionalización llevados a cabo por a través del tejido organizativo.

En el cuadro 1 se expone un cuadro sinóptico que recoge la relación entre tipo de transacción y tipo de sociedad, así como con el tipo de juego y de legitimidad predominante.

Las sociedades tradicionales deben su cohesión social a rutinas no alteradas durante largo tiempo. Prevalece lo normal, la norma, por encima de todo. Ello da lugar a mapas cognitivos acerca del mundo y la sociedad relativamente rígidos. Lo nuevo es rechazado casi por sistema. Sólo hay una forma de hacer las cosas y es invariable. Todos los individuos pueden ganar con este tipo de sociedad si tenemos en cuenta que el mundo tiene un sentido unívoco para ellos. Están protegidos por el padre común, el conjunto de símbolos que los protegen de lo desconocido, los dioses, los mitos. Los rituales vehiculan la acción social en casi todas sus facetas y los transgresores son duramente sancionados. La autoridad se concentra en elites de tipo tradicional legitimadas por su propia permanencia en la cúspide de la pirámide social $^{7}$. La cohesión social es muy alta y lo que es considerado como normal abarca una estrecha variedad de opiniones y formas de conducirse. Esta homogeneidad cultural es a la vez causa y efecto de una diferenciación de roles relativamente sencilla y altamente jerarquizada.

La sociedad en estado naciente ${ }^{8}$ se caracteriza por predominar en ella las transacciones niño-niño. Prevalece lo emocional y la innovación. El entusiasmo contagia al colectivo de forma que ni las normas ni los razonamientos consiguen aplacar sus 
ánimos. El mejor ejemplo es el estado de conciencia colectivo del fervor revolucionario. Todo es posible. Un mundo nuevo se abre ante los ojos de los actores sociales, que lo miran con ojos infantiles. Todo comienza de nuevo. Se trata de un mundo por descubrir, lleno de encanto. Es el estado de carisma a que se refiere Weber. Es la forma en que cambian las sociedades tradicionales. Muerto el anterior padre, normalmente personificado en una figura paterna, (y rodeado de una simbología, instituciones y organizaciones) mueren las viejas normas y los niños de los individuos campan libremente hasta que se comienzan a establecer nuevas rutinas y un nuevo orden social. Los niños de los individuos entronizan un nuevo padre con el cual se identificarán, de forma que con el tiempo estaremos de nuevo ante una sociedad de corte tradicional.

Así, las sociedades tradicionales cambiarían de una forma cíclica, como un individuo ciclotímico, que alterna fases de hiperactividad (el niño) con fases de rigidez y pesadumbre (el padre). El carisma sería el motor del cambio en estas sociedades, en mayor medida que en las demás, ya que permite pasar de la transacción complementaria padre-padre a la niño-niño gracias a un líder carismático en el cual se expresan de forma extrema el conflicto entre el padre y el niño, entre tradición y deseo. Esto hace que conecte fácilmente con la mayoría de la sociedad, cuyo padre es casi idéntico.

Las sociedades tradicionales tienen otras formas de mantener el equilibrio a través de cambios de tipo ritual. Los más típicos consisten en establecer fechas y días señalados para liberar las pulsiones reprimidas, catarsis colectivas ritualizadas, como el carnaval, las orgías, pequeños espacios para resarcir al niño y de esta forma liberar tensión social. En esos días el orden se subvierte o invierte y cada uno da rienda suelta a sus deseos más oscuros (a la luz de la normalidad vigente). Después, todo sigue siendo igual que antes.

También puede predominar este tipo de transacciones no en forma de relaciones de complicidad sino de abierta competencia. Algunas sociedades alientan a los individuos a competir entre sí, lo que les resta energías para emplearlas en relaciones de complicidad. Probablemente es la forma en que se manifiesta el carisma en las sociedades occidentales actuales, de forma que no constituye ninguna amenaza para el sistema siempre y cuando se respeten unas normas mínimas para el funcionamiento del mercado. De ser posible la existencia de sociedades totalmente competitivas, en la línea de la mitología liberal al respecto, también nos encontraríamos con una sociedad caótica, sin normas. Es de notar, sin embargo, la gran capacidad para el cambio y la innovación que presenta este tipo de configuración social, basado en la competencia.

Las sociedades modernas ${ }^{9}$ se estructuran en torno a las transacciones entre adultos, mediante contratos ${ }^{10}$. Predomina el cálculo racional de costes y beneficios. La cohesión social se basa en códigos escritos, consensuados por la mayoría de los individuos (directamente o a través de representantes). El criterio de normalidad está mucho más disperso que en las sociedades tradicionales, como está más desarrollada la diferenciación de roles. Esto permite una mayor variedad de opiniones y comportamientos y la mayor posibilidad de realizar innovaciones, acelerando el 
ritmo del cambio social, que acaba siendo la norma. Los cambios son pactados, aunque hay que recordar que el adulto tiene a la vez algo de niño y de padre y que pueden surgir conflictos entre los deseos de algunos actores sociales y lo considerado como normal entre aquellos y los deseos de otros actores sociales. Es una sociedad en la que es más posible la previsión y el cálculo de probabilidades. Pero, por lo general, los cambios que tienen lugar son de tipo 1 o dentro del sistema. La capacidad de homeostasis es muy alta y no son necesarios los altibajos cíclicos de las sociedades tradicionales. El comportamiento y el pensamiento son más racionales, pero no está del todo claro que ello excluya la tradición y el carisma. En todo caso, pudiera ser que el carisma estuviera más repartido, que los individuos tuvieran más oportunidades para ejercer sus deseos y menos necesidades de rituales catárticos que para restablecer el equilibrio. El padre, la norma, se haya sometida a continua revisión ${ }^{11}$. La propia norma es producto de la razón, o, como diría Giddens, las tradiciones dentro de las sociedades modernas serían tradiciones 'modernas'.

El último tipo de sociedad, basada en transacciones complementarias sería la sociedad de tipo autoritario. Suele darse como reacción al fracaso de pasar de una sociedad tradicional a una moderna. En ausencia de adultos fuertes, la «muerte del padre» sin su sustitución por otro puede angustiar tanto a los niños que estos busquen una figura protectora y omnipotente a la que someterse. El miedo al caos, la anomia y el desconcierto resultante de no pasar de una sociedad donde domina una transacción estable a otra de la misma naturaleza, genera este tipo de respuestas.

Finalmente, hay que hacer referencia a las transacciones cruzadas. Son propias de sociedades en conflicto. Podríamos distinguir entre conflictos violentos o choques culturales y conflictos controlados.

Cuadro 1. - Relación entre tipo de transacción predominante, tipo de sociedad y otras características

\begin{tabular}{|c|c|c|c|c|c|c|}
\hline $\begin{array}{l}\text { TIPO DE } \\
\text { TRANSACCIÓN }\end{array}$ & \multicolumn{4}{|c|}{ COMPLEMENTARIA } & \multicolumn{2}{|c|}{ CRUZADA } \\
\hline $\begin{array}{l}\text { TRANSACCIÓN } \\
\text { PREDOMINANTE }\end{array}$ & P-P & $\mathrm{N}-\mathrm{N}$ & A-A & $\mathrm{P}-\mathrm{N}$ & $\begin{array}{l}\text { P-N } \\
\text { N-P }\end{array}$ & $\begin{array}{l}\text { P-N } \\
\text { A-A }\end{array}$ \\
\hline $\begin{array}{l}\text { TIPO DE SOCIE- } \\
\text { DAD }\end{array}$ & $\begin{array}{l}\text { TRADI- } \\
\text { CIO- } \\
\text { NAL } \\
\end{array}$ & \begin{tabular}{|l|} 
ESTADO \\
NA- \\
CIENTE
\end{tabular} & $\begin{array}{l}\text { MO- } \\
\text { DERNA }\end{array}$ & $\begin{array}{l}\text { AUTORI- } \\
\text { TARIA }\end{array}$ & $\begin{array}{l}\text { POLARIZA- } \\
\text { CION } \\
\text { CHOQUE } \\
\text { CULTURAL }\end{array}$ & EN CAMBIO \\
\hline $\begin{array}{l}\text { TIPO DE } \\
\text { INTERACCIÓN }\end{array}$ & $\begin{array}{l}\text { CON- } \\
\text { FORMI- } \\
\text { DAD }\end{array}$ & $\begin{array}{l}\text { COMPLI- } \\
\text { CIDAD } \\
\text { COMPE- } \\
\text { TENCIA } \\
\end{array}$ & $\begin{array}{l}\text { INTER- } \\
\text { CAMBIO }\end{array}$ & $\begin{array}{l}\text { COER- } \\
\text { CIÓN }\end{array}$ & $\begin{array}{l}\text { CONFLICTO } \\
\text { ABIERTO }\end{array}$ & $\begin{array}{l}\text { CONFLICTO } \\
\text { CONTROLA- } \\
\text { DO }\end{array}$ \\
\hline TIPO DE JUEGQ & SUMA 1 & SUMA 1 & SUMA 1 & SUMA 0 & \begin{tabular}{|l|} 
SUMA NO \\
O NEGATI- \\
VA \\
\end{tabular} & $\begin{array}{l}\text { SUMA NO 0 } \\
\text { NEGATIVAA } \\
\text { SUNA } 1\end{array}$ \\
\hline $\begin{array}{l}\text { PPIO. DE } \\
\text { LEGITIMACIÓN }\end{array}$ & $\begin{array}{l}\text { TRADI- } \\
\text { CIÓN }\end{array}$ & $\begin{array}{l}\text { CARIS- } \\
\text { MA }\end{array}$ & $\begin{array}{l}\text { RAZÓN- } \\
\text { LEY }\end{array}$ & & & \\
\hline
\end{tabular}


En el conflicto violento, se da un enfrentamiento entre dos padres, entre dos normas o formas de entender la vida. No hay conciliación posible porque el padre es rígido. Todo comienza por la agresión sufrida por el niño de unos (sus deseos y aspiraciones) por parte del padre de otro (la norma social de otro colectivo). Esto hace reaccionar al padre del segundo que intenta devolver el golpe respaldado por su universo simbólico propio. No se puede negociar en este contexto. Es la situación propia de los choques entre dos culturas distintas (dos padres). También es lo típico en las situaciones de polarización social dentro de una misma cultura. Si las condiciones de vida de los distintos colectivos que conviven dentro de una colectividad difieren de tal forma que comienzan a elaborar sus pautas culturales propias (generalmente por contraposición a la establecida) pueden surgir poderosos conflictos, ya sea de clase o de nacionalidad, dificultando la cooperación al interior del sistema. Por último habría que hablar del conflicto controlado. Se trata de una transacción excluyente que se caracteriza por responder con una conciliación adultoadulto frente a una confrontación padre-niño. El adulto es capaz de ver el niño, el padre y el adulto del otro, desde el momento en que es capaz de ver los suyos propios y conciliarlos. De esta forma puede intentar «llamar» al adulto del otro, llamarlo a la reflexión, al razonamiento, haciendo que se haga un hueco entre las normas culturales rígidas del padre y los deseos compulsivos del niño. Esto limaría las asperezas, tendiendo un puente de plata al enemigo. El conflicto sigue ahí, pero los actores sociales conviven con él y puede incluso ser un motor de cambio en los dos sentidos. Los cambios generados a través de la mediación social tienden a respetar los límites (padre) del sistema. Tienden, por lo tanto, a configurar cambios de tipo 1.

Para comprender mejor como intervienen los diversos elementos de la estructura tripartita propuesta para afrontar los distintos niveles de análisis, habría que hacer referencia a la teoría del interactor social desarrollada por Berger, Eyre y Zelditch (1989), según la cual los actores pueden ser individuos pero también organizaciones o Estados. Según estos autores, la unidad básica de análisis es el actor en su situación. Se ha considerado también el esquema multidimensional propuesto por Alexander (1982), estructurado en torno a dos ejes: individualcolectivo y acción instrumental-normativa. Para el modelo aquí propuesto, el actor social (individual o colectivo) se adapta a la realidad externa en función de la información que recibe de ésta y de sus esferas normativa (padre), expresiva (niño) e instrumental (conocimiento de experiencias pasadas acumuladas y procesadas por el adulto). La estructuración social se produce mayormente a través de outputs exteriorizados desde la esfera normativa y el cambio social desde outputs emitidos desde la esfera expresiva. La mediación social cumple la función de transmitir y conciliar o hacer compatibles unos y otros. Un mayor grado de conocimiento por parte del actor social logrado a través de la mediación social le brinda mayores grados de libertad en la medida en que es capaz de comprender y explicar tanto la realidad externa como su propia realidad. Se trata de la consecución de una mayor 
capacidad para variar el repertorio de respuestas socialmente aceptables a un estímulo dado $^{12}$. Trasladando este esquema al sistema social, la capacidad de autopoiesis es mayor en la medida en que sea mayor la capacidad para procesar la información proviniente de la realidad exterior (entorno) e interior al sistema. El conocimiento y evaluación de la realidad imprime mayor racionalidad a la acción, que es principalmente significativa o expresiva en los grupos pequeños, instrumental en las organizaciones formales e histórica en las sociedades nacionales. Los sistemasindividuo, de interacción y social serían autopoiéticos en la terminología de Luhmann (1984/1998) en la medida en que existe un elemento racional que procesa y da unidad y sentido tanto a realidad externa como a los propios elementos normativos y expresivos del sistema. Este proceso de racionalización es llevado a cabo por la parte adulta del sistema individuo y se proyecta en forma de acción significativa dentro del grupo social, de acción instrumental o mediación, dentro de la organización y de acción histórica en el nivel macro-social o institucional.

Dentro de los grupos, la acción (dotada de significado para los individuos) tiene que conciliar las rutinas del grupo con las motivaciones de los distintos individuos que lo componen. Dentro de las organizaciones, la acción tiende a ser más instrumental a la vez que formalizada, conciliando los objetivos de los miembros de la misma con las normas vigentes. En el plano macro-social el resultado de la interacción entre tradición y el carisma individual conduce a la acción histórica, significativa para un importante colectivo y con trascendencia en el curso de la sociedad (ver cuadro 2).

Como se puede observar, la mayor encrucijada entre cambio y estructura, entre micro y macro, está en la mediación llevada a cabo desde y dentro de las organizaciones, mayormente centrada en la acción instrumental.

Cuadro 2. - Niveles de análisis, elementos y procesos de estructuración y cambio social

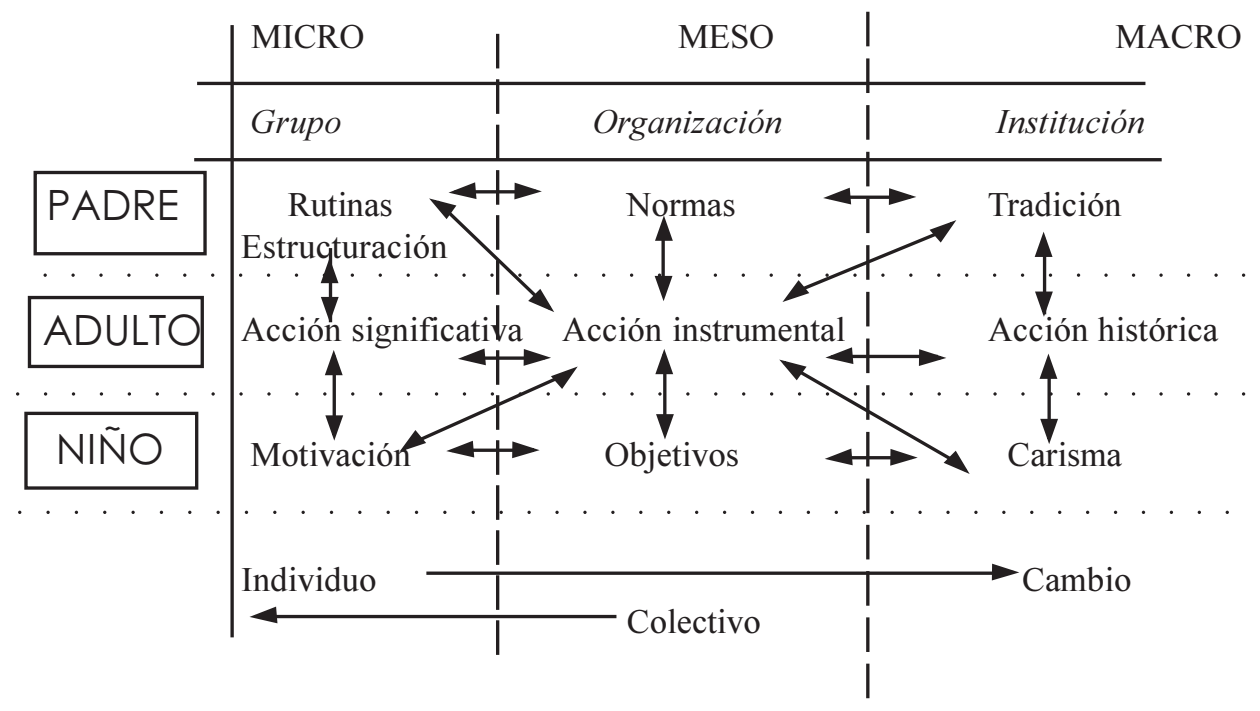


Finalmente, es posible explicar las secuencias de cambio social más probables en función de las transacciones más frecuentes dentro de un colectivo. La mayor probabilidad de paso de un tipo de transacción a otra está en relación inversa con los cambios realizados en el tipo de transacción. Es más probable pasar de un tipo de transacción a otra en la medida en que se mantenga constante uno de los dos términos de la misma. Es más fácil pues pasar de una sociedad de corte tradicional (P-P) a uno de corte burocrático (P-A) o autoritario (P-N) que a pasar directamente a uno de corte plenamente moderno (A-A). Sólo hace falta variar uno de los dos términos de la interacción. Así, lo más frecuente es que para pasar de un estado típico-ideal a otro se tenga que pasar por una fase intermedia de transición (ver cuadro 3). La transición suele consistir en un contexto social dominado por los juegos de suma -1 (solución estratégica del conflicto) donde los actores sociales buscan alianzas a costa de perder parte de su beneficio. Esto puede llevar a otro esquema de transición o a un estado social típico con distintas características que el anterior, pudiendo evolucionar hacia contextos sociales dominados por juegos de suma 1 o por juegos de suma 0 (solución autoritaria al conflicto). El tránsito de la sociedad tradicional hacia otros tipos de sociedad se suele operar a través de un proceso de racionalización asociado al surgimiento de una burocracia, con lo cual se sustituye la prevalencia de las transacciones P-P por las de tipo P-A. Un fracaso en la introducción del factor racionalizador e instrumental (adulto) en las transacciones de corte tradicional (P-P), puede conducir a través de una burocratización y racionalización fallida a una sociedad de corte autoritario (P-N). Hemos comprobado estos procesos fallidos y sus consecuencias tanto en países europeos como en países actualmente en vías de desarrollo. Por el contrario, la pérdida de importancia del factor normativo (padre) en las transacciones y la ganancia del factor instrumental puede conducir a una sociedad de corte moderno donde prevalecen las transacciones A-A. El ejemplo más claro sería el de las sociedades escandinavas. Si lo que ocurre es que hay una menor constricción de los impulsos individuales, donde una elite gestiona o arbitra el conflicto en un contexto normativo mínimo estaríamos ante una sociedad de corte revolucionario-liberal, donde todo vale ${ }^{13}$. Sería el caso de los Estados Unidos de América. Los dos tipos de transición presentan las mismas probabilidades de evolucionar hacia un tipo-ideal moderno como hacia uno de corte autoritario en función del desarrollo del elemento racional en las transacciones entre individuos, grupos y organizaciones $^{14}$. 
Cuadro 3. - Matriz de procesos de cambio de estado social ${ }^{15}$

\begin{tabular}{|c|l|l|l|l|}
\hline ESTADO & \multicolumn{2}{|l|}{$\begin{array}{l}\text { ESTADO SOCIAL Y TIPO DE TRASACCIÓN } \\
\text { PREDOMINANTE }\end{array}$} & $\begin{array}{l}\text { TIPO DE JUEGO } \\
\text { Y DE SOLUCIÓN } \\
\text { CONFICTO }\end{array}$ \\
\hline $\begin{array}{l}\text { TÍPICO- } \\
\text { IDEAL }\end{array}$ & $\begin{array}{l}\text { TRADICIONAL } \\
\text { (P-P) }\end{array}$ & $\begin{array}{l}\text { MODERNA } \\
(\mathrm{A}-\mathrm{A})\end{array}$ & $\begin{array}{l}\text { E. NACIENTE } \\
\text { (N-N) }\end{array}$ & $\begin{array}{l}\text { SUMA 1 } \\
\text { (ÓPTIMA) }\end{array}$ \\
\hline $\begin{array}{l}\text { TRANSITO- } \\
\text { RIO O MIXTO }\end{array}$ & $\begin{array}{l}\text { BUROCRÁTICO } \\
\text { (P-A) }\end{array}$ & $\begin{array}{l}\text { REVOLUCIONARIO } \\
\text { (N-A) }\end{array}$ & $\begin{array}{l}\text { SUMA - 1 } \\
\text { (ESTRATÉ- } \\
\text { GICA) }\end{array}$ \\
\hline $\begin{array}{l}\text { TÍPICO- } \\
\text { IDEAL }\end{array}$ & \multicolumn{2}{|c|}{$\begin{array}{l}\text { AUTORIATARIO } \\
\text { (P-N) }\end{array}$} & $\begin{array}{l}\text { SUMA 0 } \\
\text { (AUTORI- } \\
\text { TARIA) }\end{array}$ \\
\hline
\end{tabular}

\section{CONCLUSIONES Y DISCUSIÓN}

El modelo propuesto presenta la posibilidad de medir el nivel de cambio o estructuración social en función de variables únicamente sociales a través de la medición en el tiempo de los flujos transaccionales entre los individuos dentro de un sistema social. Mediante la medición de dichos flujos dentro del sistema transaccional, o dicho de otra forma, mediante la medición de la frecuencia e intensidad de los tipos de flujos que operan a través de este mecanismo que conecta individuos con sistema social, se podrían diagnosticar y predecir la viabilidad de una tendencia de cambio en un sistema social determinado en sus diferentes niveles. Esto podría dotar de herramientas operativas y con calado teórico a los profesionales que ejerzan la labor de mediador social en cualquiera de dichos niveles. Todo ello hace posible someter el cambio social a diagnóstico, planificación y evaluación desde una perspectiva meramente social, observable a corto plazo en cada uno de los niveles del sistema social. Los cambios a largo plazo no se observan sólo a través del cambio del tipo de transacción predominante pero los cambios a corto plazo sí. Algo de vital importancia cuando tratamos de evaluar los resultados que para el cambio social ha tenido una intervención puntual.

Las limitaciones del modelo son las propias de explicar lo social desde lo social, manteniendo las variables externas o del entorno en condición de ceteris paribus. Los factores tecnológicos, económicos, políticos, culturales y ecológicos aparecen como externos al sistema. No hay manera de explicar cambios de sistema si no hay cambios exteriores derivados del choque con otros sistemas sociales, o de cambios tecnológicos, económicos, ecológicos o estratégicos que den pie a la quiebra del nivel institucional. No aparece clara la posibilidad de cambio de sistema 
desde dentro si no es con dicha presión exterior. Tan sólo se plantea la posibilidad de maximizar al límite las posibilidades de cambio dentro del sistema o, dicho en términos marxistas, de acelerar la historia desde la superestructura pero sin alterar las condiciones objetivas o infraestructurales para llevar acabo un cambio de sistema.

Otra cuestión pendiente es la consideración de la red social como un sistema autopoiético o como un mero mecanismo de transmisión de información entre sistemas.

Queda también por desarrollar (y será objeto de próximos escritos) el modelo complejo de relación entre distintos sistemas sociales como interactores, a nivel grupal, organizacional e institucional, ya que se pueden considerar como elementos de una red tanto a individuos como a colectivos. Dentro de este esquema de relaciones complejas sería además deseable desarrollar una teoría aplicable de la mediación social que haga en cierto modo previsibles y verificables los resultados de la misma.

Finalmente, queda por desarrollar una doble validación empírica (interna y externa) del modelo propuesto. La primera, a través de la construcción de un modelo informatizado de simulación social con parámetros y escenarios virtuales; la segunda, a través de su aplicación con parámetros y escenarios reales, en los cuales se aplique un método de diagnostico, intervención y evaluación basado en el modelo propuesto a través del desarrollo de los pertinentes instrumentos de medición. 


\section{NOTAS}

${ }^{1}$ LUHMANN, N., (1996) p.31, dirá como frase-resumen de la teoría sistémica de PARSONS: 'Action is system'.

${ }^{2}$ El problema abordado por OLSON consiste en cómo explicar la acción colectiva y la producción de bienes públicos partiendo del supuesto de que todos los individuos se comportan de manera racional, tratando de obtener beneficios al menor coste posible. ${ }^{3}$ Sobre el sospechoso abandono que de la cuestión emocional han mostrado las ciencias sociales y de las consecuencias para el estudio de la comunicación hay una clara referencia en un artículo reciente de BERICAT (1999).

${ }^{4}$ En España, el modelo y las aplicaciones de esta teoría han sido desarrolldas por José María ROMÁN y otros autores (1994).

${ }^{5}$ TÖNNIES, F. (1935/1979) procede de forma semejante al utilizar el concepto de estado social como unidad de análisis.

${ }^{6}$ Por output se entiende una salida da energía o información hacia el entorno del sistema.

${ }^{7}$ Se trata del sistema de dominación tradicional argumentado por Max WEBER $(1922 / 1984)$.

${ }^{8}$ Término empledo por Francesco ALBERONI: "El estado naciente es una tentativa de rehacer el mundo...una exploración de lo posible a partir de lo imposible, de realizar le estado paradisíaco en la tierra." (1988), p.65.

${ }^{9} \mathrm{El}$ término moderno es empleado de manera restringida en referencia a aquellos contextos sociales donde la racionalidad impera en las transacciones sociales. Habría sociedades de corte moderno (en un sentido más extenso del término) en los tipos mixtos o de transición (ver cuadro 3).

${ }^{10}$ Esto era defendido en cierta forma por Tönnies en su distinción entre estados sociales de «Comunidad» $\mathbf{y}$ «Asociación» (1935/1979).

${ }^{11}$ Se correspondería con el modo de dominación legal-racional de Max WEBER.

${ }^{12}$ La ruptura del arco reflejo estímulo-respuesta que planteaba MEAD (1972), puede pues adquirir una mayor variedad de manifestaciones que la interiorizada inicialmente, lo cual nos presenta un individuo con mayores grados de libertad.

${ }^{13}$ No hay que olvidar al respecto que MARX consideraba a la burguesía como la clase más revolucionaria de la Historia (1872/1987).

${ }^{14}$ También hay que considerar que se consideran tipos de transición o mixtos por no tratarse de tipos puros, pero ello no significa que no puedan ser tan estables como los otros estados sociales.No se deb confundir en ningún caso estado de transición con estado transitorio.

${ }^{15}$ El concepto de estado social aquí utilizado consiste en el mismo que utiliza TÖNNIES (1935/1979) extrapolado a los términos del análisis transaccional, lo cual aumenta las combinaciones posibles al considerar no sólo dos tipos de voluntades esenciales (orgánica y arbitraria) sino tres (padre, adulto y niño). 


\section{BIBLIOGRAFÍA}

ALEXANDER, J., (1982): Theoretical Logic in Sociology, vol. 1, Positivism, Presuppositions, and Current Controversies, University of California Press, Berkeley. ALBERONI, F., (1988): Enamoramiento y amor, Gedisa, Barcelona.

AXELROD, R., (1984/1986): La evolución de la cooperación, Alianza, Madrid. BERGER, J.; EYRE, D. y ZELDITCH, M., (1989): "Theoretical Structuresand the Micro/Macro Problem". En BERGER, J., ZELDITCH, M., y ANDERSON, B., (eds.): Sociological Theories in Progress: New formulations, Sage, Newbury Park, California, 11-32.

BERICAT ALASTUEY, E., (1999): "El contenido emocional de la comunicación en la sociedad de riesgo", REIS, num.87, pp. 221-254.

BERNE, E., (1961/1976): El análisis transaccional en psicoterapia, Psique, Buenos Aires. DURKHEIM, E., (1894/1982): Las reglas del método sociológico, La Pléyade, Buenos Aires.

GERTH, H. y MILLS, C.W., (1984): Carácter y estructura social, Paidós, Barcelona. GIDDENS, A., (1984): The Constitution of Society: Outline of Theory of Structuration, University of California Press, Berkeley.

GURTVITCH, G., (1964): The Spectrum of Social Time, Dordecht, P.B.: D. Reidel. BARRINGTON, M., (1976): Los orígenes sociales de la dictadura y la democracia, Península, Barcelona.

LUHMANN, N., (1996): Introducción a la teoría de sistemas, Universidad Iberoamericana, México.

(1984/1998): Sistemas sociales, Anthropos, Barcelona.

MARX, K. Y ENGELS, F., (1872/1987): El manifiesto comunista, Alba, Madrid. MAYER, A.C., (1966/1990): "La importancia de los cuasi-grupos en las sociedades complejas". En WOLF, E., MITCHELL, C. y otros, Antropología Social de las sociedades complejas, Alianza Universidad, Madrid.

MEAD, H.G., (1972): Espíritu, persona y sociedad, Paidós, Buenos Aires.

MERTON, R., (1992): Teoría y estructura sociales, F.C.E. ,México.

MILLS, C.W.,(1959/1987): La imaginación sociológica. México. Fondo de Cultura. OLSON, M., (1971/1992): La lógica de la acción colectiva, Limusa, México. PARSONS, T., (1959/1966): El sistema social, Revista de Occidente, Madrid.

(1961/1968): El sistema de la acción social, Guadarrama, Madrid.

RITZER, G., (1979): “Toward an Integrated Sociological Paradigm”. En SNIZEK et al. (eds.): Contemporary Issues in Theory and Research, Greenwood Press, Westport, Connecticut, pp. 25-46.

ROMÁN, J.M., (et al.) (1994): Análisis transaccional. Modelo y aplicaciones, Amaru, Salamanca.

TÖNNIES, F., (1935/1979): Comunidad y asociación, Península, Barcelona. WEBER, M., (1922/1984): Economía y sociedad, F.C.E., México. 